\title{
A PEG-Fmoc conjugate as a nanocarrier for paclitaxel
}

\author{
Peng Zhanga,b,c, Yixian Huang ${ }^{a, b, c}$, Hao Liud ${ }^{d}$ Rebecca Marquez ${ }^{d}$, Jianqin Lua \\ Zhao $^{b}$, Xiaolan Zhang ${ }^{a, b, c}$, Xiang Gao ${ }^{a, b, c}$, Jiang Li ${ }^{a, b, c}$, Raman Venkataramanan ${ }^{b}$, Liang \\ $\mathbf{X u}^{\mathrm{d}}$, and Song $\mathbf{L i}^{\mathrm{a}, \mathrm{b}, \mathrm{c},{ }^{*}}$ \\ ${ }^{a}$ Center for Pharmacogenetics, University of Pittsburgh, Pittsburgh, PA, 15261, USA \\ bDepartment of Pharmaceutical Sciences, School of Pharmacy, University of Pittsburgh, \\ Pittsburgh, PA, 15261, USA \\ 'University of Pittsburgh Cancer Institute, University of Pittsburgh, Pittsburgh, PA, 15261, USA \\ dDepartments of Molecular Biosciences and Radiation Oncology, University of Kansas Cancer \\ Center, University of Kansas, Lawrence, KS, 66045, USA
}

\begin{abstract}
We report here that a simple, well-defined, and easy-to-scale up nanocarrier, $\mathrm{PEG}_{5000}$-lysyl-(aFmoc- $\varepsilon$-t-Boc-lysine) ${ }_{2}$ conjugate (PEG-Fmoc), provides high loading capacity, excellent formulation stability and low systemic toxicity for paclitaxel (PTX), a first-line chemotherapeutic agent for various types of cancers. 9-Fluorenylmethoxycarbonyl (Fmoc) was incorporated into the nanocarrier as a functional building block to interact with drug molecules. PEG-Fmoc was synthesized via a three-step synthetic route, and it readily interacted with PTX to form mixed nanomicelles of small particle size $(25-30 \mathrm{~nm})$. The PTX loading capacity was about $36 \%$, which stands well among the reported micellar systems. PTX entrapment in this micellar system is achieved largely via an Fmoc/PTX $\pi-\pi$ stacking interaction, which was demonstrated by fluorescence quenching studies and ${ }^{13} \mathrm{C}-\mathrm{NMR}$. PTX formulated in PEG-Fmoc micelles demonstrated sustained release kinetics, and in vivo distribution study via near infrared fluorescence imaging demonstrated an effective delivery of Cy5.5-labled PTX to tumor sites. The maximal tolerated dose for PTX/PEG-Fmoc (MTD > $120 \mathrm{mg} \mathrm{PTX/kg)} \mathrm{is} \mathrm{higher} \mathrm{than} \mathrm{those} \mathrm{for}$ most reported PTX formulations, and in vivo therapeutic study exhibited a significantly improved antitumor activity than Taxol, a clinically used formulation of PTX. Our system may hold promise as a simple, safe, and effective delivery system for PTX with a potential for rapid translation into clinical study.
\end{abstract}

\footnotetext{
(C) 2014 Elsevier Ltd. All rights reserved.

*Address correspondence to: Song Li, M.D., Ph.D., Center for Pharmacogenetics, University of Pittsburgh School of Pharmacy, 639 Salk Hall, Pittsburgh, PA 15261, Tel: 412-383-7976, Fax: 412-648-1664, sol4@ pitt.edu.

Publisher's Disclaimer: This is a PDF file of an unedited manuscript that has been accepted for publication. As a service to our customers we are providing this early version of the manuscript. The manuscript will undergo copyediting, typesetting, and review of the resulting proof before it is published in its final citable form. Please note that during the production process errors may be discovered which could affect the content, and all legal disclaimers that apply to the journal pertain.
} 


\section{Keywords}

micelle; 9-Fluorenylmethoxycarbonyl; drug-carrier interaction; paclitaxel; drug delivery; cancer therapy

\section{Introduction}

Nanomedicine has been shown to dramatically improve the in vivo performance of various anticancer agents through modifying their physiochemical properties, pharmacokinetics, and distribution profiles [1-6]. To date, a variety of polymer- and lipid-based systems have been developed and a few of them are currently being used in the clinic [7-11]. However, it remains a challenge to develop a simple, easy-to-scale up system that provides excellent drug loading capacity and formulation stability.

As a well-developed delivery system, micelles are attractive due to the ease of preparation, and small sizes that can contribute to a reduced rate of elimination from circulation and enhanced accumulation at solid tumors with leaky vasculature based on the enhanced penetration and retention (EPR) effect [12, 13]. Most of the current micellar systems are composed of two distinct domains, one being hydrophilic and the other hydrophobic, and drug loading is solely based on the interactions of their hydrophobic domains with the poorly water-soluble drugs [14-16]. While working well for highly hydrophobic/lipophilic agents, these systems exhibit limited effectiveness for drugs with moderate hydrophobicity due to limited drug-carrier compatibility.

Recent studies have highlighted the benefits of introducing other drug-interactive domains into the conventional micellar systems to improve the drug loading capacity and formulation stability through introduction of additional mechanisms of carrier/drug interactions. For example, some studies have demonstrated that inclusion of a hydrotropic domain or entire drug molecule such as doxorubicin can effectively improve the performance of several polymeric systems with respect to drug loading capacity and colloidal stability of drugloaded micelles [17-21].

We have recently developed a new concept that introduction of a drug-interactive domain at the interfacial region represents an effective strategy to improve the compatibility between lipid-core micelles and the hydrophobic drugs. Among several drug-interactive domains tested, 9-Fluorenylmethoxycarbonyl (Fmoc) was shown to have an unusual propensity in interacting with many types of agents of diverse structure and water solubility [22]. After interfacially modified with Fmoc groups, polyethylene glycol (PEG)-lipopeptides are active in formulating a panel of dissimilar drugs, ranging from paclitaxel (PTX), steroids, xanthene- and porphyrin-based photodynamic agents, to hydrophobic peptide drugs, with significant improvements in both drug loading capacity and drug retention [23]. These data strongly suggest that Fmoc qualifies as a "formulation chemophor", exhibiting a potent activity in interacting with various pharmaceutical agents and thus a capability of improving carrier-drug compatibility. 
In general, it is believed that a large hydrophobic domain such as a lipid chain or hydrophobic polymer is necessary to construct micelle-forming surfactants, and indeed, Fmoc-containing PEG-lipid conjugates were more effective than the counterparts without a lipid motif in formulating a number of hydrophobic agents. Interestingly, a PEG-Fmoc conjugate without a lipid motif, PEG $_{5000}$-lysyl-(a-Fmoc- $\left.\left.\varepsilon-t-B o c-l y s i n e\right)\right)_{2}$ (PEG-Fmoc), was found to be highly effective in solubilizing PTX. More surprising is the finding that PEGFmoc was significantly more effective than the counterpart with a lipid motif in formulating PTX. This study is focused on characterization of PEG-Fmoc as a simple and effective micellar formulation for PTX. The potential mechanism involved in the drug/carrier interaction between PEG-Fmoc and PTX is also investigated, which may shed insights into the future development of further improved nanocarrier for therapeutic agents.

\section{Materials and methods}

\subsection{Reagents}

Paclitaxel (PTX, >99\%) was purchased from TSZ Chem (MA, USA). Docetaxel (DTX, $>99 \%$ ) was obtained from LC Laboratories (MA, USA). a-Fmoc- $\varepsilon$-Boc-lysine, di-Boclysine, N, N'-dicyclohexylcarbodiimide (DCC), N-hydroxysuccinimide (NHS), trifluoroacetic acid (TFA), and triethylamine (TEA) were obtained from Acros Organic (NJ, USA). Monomethoxy $\mathrm{PEG}_{5000}$, monomethoxy $\mathrm{PEG}_{550}$, cholesterol (CHOL), 4dimethylaminopyridine (DMAP), ninhydrin, and other unspecified chemicals were all purchased from Sigma-Aldrich (MO, USA). Dulbecco's phosphate buffered saline (DPBS), Dulbecco's Modified Eagle's Medium (DMEM), fetal bovine serum (FBS), 100X penicillinstreptomycin solution were all purchased from Invitrogen (NY, USA). All solvents used in this study were HPLC grade.

\subsection{Synthesis of PEG ${ }_{5000}$-lysyl-(a-Fmoc-e-t-Boc-lysine) 2 (PEG-Fmoc)}

PEG-Fmoc was synthesized largely following our published method [23]. Briefly, 1 equiv. of monomethoxy PEG $_{5000}$ was mixed with excess amount of di-Boc-lysine and DCC in dichloromethane (DCM) with addition of DMAP, and the reaction was allowed at room temperature for $48 \mathrm{~h}$. The mixture was filtered and precipitated in ice-cold ether, followed

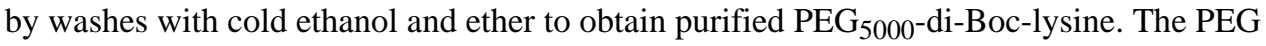
derivative was then treated with DCM/TFA $(1: 1, \mathrm{v} / \mathrm{v})$ for $2 \mathrm{~h}$ at room temperature, followed by removal of the solvent, precipitation in cold ether, and washes with cold ethanol and ether. Finally, the deprotected $\mathrm{PEG}_{5000}-\mathrm{lysine}-\mathrm{NH}_{2}$ was mixed with excess amount of aFmoc- $\varepsilon$-Boc-lysine that was pre-activated with NHS, DCC, and small amount of DMAP in DCM at $37^{\circ} \mathrm{C}$ for $4 \mathrm{~h}$. The reaction was allowed at $37^{\circ} \mathrm{C}$ till the ninhydrin test became negative, indicating the absence of free amino groups. The reaction mixture was filtered and precipitated by ice-cold ether, followed by washes with cold ethanol and ether. The obtained material was dissolved in water and filtered through a $450 \mathrm{~nm}$ filter, followed by lyophilization to yield the powder of purified PEG $_{5000}$-lysyl-(a-Fmoc-e-t-Boc-lysine) ${ }_{2}$.

\subsection{Preparation and biophysical characterization of PTX/PEG-Fmoc mixed micelles}

Thin-film hydration method was utilized for preparation of PTX/PEG-Fmoc mixed nanomicelles. PEG-Fmoc and PTX in chloroform were well mixed in a glass tube at 
designated molar ratios. A thin film of carrier/drug mixture was generated through removal of the organic solvent by a gentle stream of nitrogen. The trace amount of solvent was further removed via vacuum for $2 \mathrm{~h}$. Then the film was hydrated and suspended in DPBS by vortex to obtain a clear solution of PTX/PEG-Fmoc mixed nanomicelles. Any nonentrapped drug was removed by filtration through $450 \mathrm{~nm}$ PVDF syringe filter.

The size distribution of PTX/PEG-Fmoc mixed nanomicelles was examined by dynamic light scattering (DLS), and the zeta potential was measured via a Malvern Zeta Nanosizer. The morphology was observed by transmission electron microscopy (TEM) after negative staining. The CMC measurement was performed as reported before with pyrene as a fluorescence probe [23]. To quantify PTX in the micelles, PTX was extracted by methanol, and detected by Waters Alliance 2695-2998 high-performance liquid chromatography (HPLC) system with a RP-18 column $(250 \mathrm{~mm} \times 4.6 \mathrm{~mm})$ equipped with a UV detector at $227 \mathrm{~nm}$ at room temperature. A mixture of methanol/water (80:20, v/v) was used as mobile phase at a flow rate at $0.8 \mathrm{~mL} / \mathrm{min}$. The drug loading capacity (DLC) and efficiency (DLE) were calculated using the formula:

Drug loading capacity $\%=$ weight of drug $/$ (weight of polymer+weight of drug) $\times 100 \%$ Drug loading efficiency $\%=$ weight of drug loaded into micelles/weight of drug used $\times 100 \%$

\subsection{Effect of lyophilization/reconstitution on particle sizes}

One $\mathrm{mL}$ of PTX/PEG-Fmoc in DPBS was prepared as described above and the PTX concentration was kept at $1 \mathrm{mg} / \mathrm{mL}$. The clear solution was frozen and lyophilized overnight to obtain white powder. The powder was then reconstituted with $1 \mathrm{~mL}$ of distilled water to obtain a clear solution. Particle sizes of the PTX-loaded micelles before and after lyophilization/reconstitution were recorded via DLS using a Zetasizer.

\subsection{Differential scanning calorimetry (DSC) analysis}

A Mettler Toledo DSC was used for DSC analysis. Samples (PTX, PEG-Fmoc/PTX, and drug-free PEG-Fmoc) were accurately weighed and placed in aluminum crucibles, which were sealed using a Mettler Toledo sealer. An empty crucible was used as a reference. The temperature range was set from $25^{\circ} \mathrm{C}$ to $285^{\circ} \mathrm{C}$ with a rate at $10^{\circ} \mathrm{C} / \mathrm{min}$ using nitrogen $(50$ $\mathrm{mL} / \mathrm{min}$ ) as segment gas.

\subsection{Fluorescence quenching}

PEG $_{5000}$-lysyl-(a-Fmoc- $\varepsilon$-oleic acid-lysine $)_{2}$ (PEG-Fmoc-OA) was synthesized as reported [23]. PTX/PEG-Fmoc and PTX/PEG-Fmoc-OA mixed micelles of various PTX/carrier molar ratios were prepared in DPBS as described above, and the molar concentrations of PEG-Fmoc and PEG-Fmoc-OA carrier were kept constant for comparison. The samples were placed into a 96-well plate, and examined for the fluorescence intensity at the excitation wavelength of $270 \mathrm{~nm}$ and emission wavelength of 300-460 nm using a Synergy H1 Hybrid Multi-Mode Microplate Reader. 


\subsection{In vitro drug release kinetics}

Two $\mathrm{mL}$ of PTX/PEG-Fmoc mixed micelles in DPBS $(\mathrm{PH}=7.4)(1 \mathrm{mg}$ PTX/mL) was prepared and placed into a dialysis bag (MWCO $12 \mathrm{kDa}$, Spectrum Laboratories) that was incubated in a tank containing $200 \mathrm{~mL}$ of DPBS with $0.5 \%$ (w/v) Tween 80 under gentle shaking at $37^{\circ} \mathrm{C}$. At scheduled time points $(0,1,2,4,8,24,48$, and $72 \mathrm{~h})$, the concentration of PTX remaining in the dialysis bag was measured by HPLC as described above. Taxol formulation (6 mg PTX/mL in Cremophor EL/ethanol, 1:1, v/v) was diluted with DPBS to a final PTX concentration of $1 \mathrm{mg} / \mathrm{mL}$ and utilized as a control.

\subsection{Cell culture}

4T1-2 is a mouse metastatic breast cancer cell line. PC-3 and DU145 are two androgenindependent human prostate cancer cell lines. They were all cultured at $37^{\circ} \mathrm{C}$ in DMEM containing $10 \% \mathrm{FBS}$ and $1 \%$ penicillin-streptomycin in a humidified environment with 5\% $\mathrm{CO}_{2}$.

\subsection{In vitro cytotoxicity study}

Mouse metastatic breast cancer cell line 4T1.2, and two human prostate cancer cell lines PC-3 and DU145 were utilized to evaluate the in vitro cytotoxicity of PTX/PEG-Fmoc. Cells were seeded in 96-well plates at 1000 (4T1.2), 2000 (DU145) and 3000 (PC-3) cells per well. Twenty-four h later, cells were treated with PTX/PEG-Fmoc or Taxol with PTX concentrations ranging from 6.25 to $200 \mathrm{ng} / \mathrm{mL}$. Seventy-two h later, $20 \mu \mathrm{L}$ of 3-(4, 5dimethylthiazol-2-yl)-2,5-diphenyltetrazoliumbromide (MTT, $5 \mathrm{mg} / \mathrm{mL}$ ) was added into each well. Following incubation for $4 \mathrm{~h}$, the medium was removed and $150 \mu \mathrm{L}$ of DMSO was added to each well to solubilize the formazan crystal. The absorbance of each well was detected at $550 \mathrm{~nm}$ with a reference wavelength at $630 \mathrm{~nm}$ using a microplate reader, and cell viability was calculated based on the formula below. Untreated cells were included as a control.

$$
\% \text { cytotoxicity }=\left[1-\left(\mathrm{OD}_{\text {treat }}-\mathrm{OD}_{\text {blank }}\right) /\left(\mathrm{OD}_{\text {control }}-\mathrm{OD}_{\text {blank }}\right)\right] \times 100 \%
$$

\subsection{Animals}

Female BALB/c mice (6 to 8 weeks) were purchased from Charles River (Davis, CA), and male nude mice (6 to 8 weeks) were purchased from Harlan (Livermore, CA). All animals were housed under pathogen-free conditions according to AAALAC guidelines. All animalrelated experiments were performed in full compliance with institutional guidelines and approved by the Animal Use and Care Administrative Advisory Committee at the University of Pittsburgh.

\subsection{Maximum tolerated dose (MTD) study}

$\mathrm{BALB} / \mathrm{c}$ mice were randomly divided into seven groups $(\mathrm{n}=4)$ and i.v. administered with PTX/PEG-Fmoc (30, 50, 75, 100, and $120 \mathrm{mg}$ PTX/kg) or Taxol (15, 20, and $25 \mathrm{mg}$ PTX/ $\mathrm{kg}$ ). Mice were then followed for 10 days for survival and changes in body weight. The MTD was determined at a maximal dose of PTX that leads to neither animal death nor 
significant changes in the general appearance or greater than $15 \%$ of body weight loss over the entire experimental period.

\subsection{Fluorescence optical imaging}

Near infrared (NIR) fluorescence imaging was performed to investigate the in vivo biodistribution of PTX/PEG-Fmoc mixed nanomicelles using PTX labeled with Cy5.5, a near infrared fluorescence dye. Two hundred $\mu \mathrm{L}$ of PTX-Cy5.5/PEG-Fmoc mixed nanomicelles (0.2 PTX-Cy5.5 mg/mL) were i.v. injected into SCID mice bearing PC-3 xenografts. At scheduled time points, the mice were anesthetized and scanned with a Carestream Molecular Imaging's In-Vivo Imaging FX Pro, using a $60 \mathrm{sec}$ exposure time with the excitation at $630 \mathrm{~nm}$ and the emission at $700 \mathrm{~nm}$. At the end of the imaging study, all the mice were sacrificed and the major organs and tumors were excised for ex vivo imaging.

\subsection{In vivo therapeutic efficacy}

A syngeneic mouse breast cancer model (4T1.2) was established via s.c. inoculation of 2 $\times 10^{5}$ of $4 \mathrm{~T} 1.2$ cells into the right flank of female BALB/c mice, and the treatments were initiated when the tumor size reached $\sim 50 \mathrm{~mm}^{3}$. Mice were divided randomly into five groups $(\mathrm{n}=5)$ and received i.v. injection of saline, Taxol (10 mg PTX/kg), and PTX/PEGFmoc $(10,20$, and $40 \mathrm{mg}$ PTX/kg), respectively. Tumor volumes were measured by a caliper and calculated based on the formula: $\left(\mathrm{L} \times \mathrm{W}^{2}\right) / 2$, where $\mathrm{L}$ is the longest and $\mathrm{W}$ is the shortest tumor diameters $(\mathrm{mm})$. The data were presented as relative tumor volume (RTV, the tumor volume at a given time point divided by the tumor volume prior to first treatment). Mice were sacrificed when tumors developed ulceration. Changes in body weights of all mice were also monitored during the entire course of treatment to evaluate the potential toxicity of the formulations. Tumors were harvested at the completion of the experiment and tumor weights were measured. Tumor growth inhibition rate (IR) was calculated as: 1(mean tumor weight of PTX treated group/mean tumor weight of saline treated group) * $100 \%$.

\subsection{Statistical analysis}

Statistical analysis was performed with two-tailed Student's t-test between two groups. $p<$ 0.05 was considered statistically significant, and $p<0.01$ was considered highly statistically significant. One-way ANOVA was conducted to assess significance among multiple groups, followed by two-tailed Student's t-test if $p<0.05$.

\section{Results and discussion}

\subsection{Design, preparation, and characterization of PEG-Fmoc/PTX mixed micelles}

We previously developed a new lipid surfactant, PEG $_{5000}$-lysyl-(a-Fmoc- $\varepsilon$-oleic acidlysine $)_{2}$ (PEG-Fmoc-OA), and examined its utility in formulating a number of therapeutic agents of diverse structure including PTX [23]. Our data showed that PEG-Fmoc-OA was much more effective than the counterpart without Fmoc motifs $\left(\mathrm{PEG}_{5000}\right.$-di-oleoyl lysine, PEG-OA) with respect to both drug loading capacity and formulation stability. However, little was known about the molecular basis of carrier-drug interaction for our new micellar 
system. Particularly, a mode of interaction between Fmoc/PTX and its contribution to the overall performance of the new lipidic vector is largely unknown.

To clearly address this issue, we have synthesized a PEG-Fmoc conjugate without OA motifs to rule out any influence from lipid motifs whose interaction with drug also contributes to the overall performance of the new lipidic surfactant. PEG-Fmoc was readily synthesized via three steps as depicted in Fig. 1A. ${ }^{1} \mathrm{H}-\mathrm{NMR}$ spectrum of PEG-Fmoc shows signals at $3.63 \mathrm{ppm}$ attributed to the methylene protons of PEG backbone, the multiple Fmoc proton signals between 7.3-7.8 ppm, and the t-Boc signals at $1.44 \mathrm{ppm}$ (Fig. S1). The molecular weight from MALDI-TOF MS is similar to the theoretical value (6030) (Fig. S2), indicating the successful synthesis of PEG-Fmoc conjugate.

PEG-Fmoc readily formed small-sized $(25 \sim 30 \mathrm{~nm})$ micelles in aqueous solution. Negativestaining EM showed homogeneously distributed spherical particles (Fig. S3). This is different from PEG-Fmoc-OA that showed tubular morphology on EM, suggesting formation of filamentous micelles. PEG-OA was known to form spherical micelles [23]. Taken together, these data suggest that both Fmoc and lipid motifs contribute to the formation of the unique filamentous structure of PEG-Fmoc-OA.

PEG-Fmoc readily formed mixed micelles with PTX and loading of PTX had minimal effect on the size of the particles as determined by DLS (Fig. 2A). The small size and homogeneous distribution of the particles were further confirmed by negative-staining TEM as shown in Fig. 2B. Fig. 2C shows that the CMC value of PEG-Fmoc conjugate was 0.996 $\mu \mathrm{mol} / \mathrm{L}$. And ${ }^{1} \mathrm{H}-\mathrm{NMR}$ spectrum analysis showed that the signals from both PEG-Fmoc and PTX were clearly detected when they were mixed in $\mathrm{CDCl}_{3}$ (Fig. 3A). In contrast, all of the proton signals of Fmoc and PTX were drastically suppressed in deuterated water, indicating a complete encapsulation of PTX inside the core area of the self-assembled particles in aqueous solution (Fig. 3B).

Table 1 shows the drug loading capacity (DLC) at various PEG-Fmoc/PTX molar ratios. PEG-Fmoc/PTX mixed micelles could be prepared at a carrier/drug molar ratio as low as $0.25 / 1$. At this ratio, the PTX loading capacity was $\sim 36 \%$, which stands well among the reported PTX micellar formulations. Increasing the carrier/drug ratio was associated with an increase in drug loading efficiency (DLE) and an improvement in the colloidal stability of the mixed micelles in 50\% FBS/PBS. Surprisingly, PEG-Fmoc was significantly more active than PEG-Fmoc-OA that has a highest PTX loading capacity of $15.2 \%$ [23]. This is in contrast to solubilization and loading of several other drugs for which PEG-Fmoc-OA was more active than PEG-Fmoc.

\subsection{Mechanism of carrier/drug interactions}

Fmoc is known to have strong $\pi-\pi$ stacking interaction with other molecules bearing aromatic rings [24-26], which is normally stronger than the van der Waals interaction. Fmoc is likely to promote the formation of stable PEG-Fmoc micelles through the Fmoc/Fmoc interaction. The fact that PTX could be loaded into PEG-Fmoc micelles at a high loading capacity suggests that Fmoc effectively interacts with PTX. An absence of PTX endothermic melting peak in PEG-Fmoc/PTX micelles was observed in differential scanning calorimetry 
(DSC) analysis, which indicated a loss of PTX crystallinity caused by a strong carrier-PTX interaction in PEG-Fmoc/PTX micelles (Fig. 4). To further gain insight into the mechanism of drug-carrier interaction, fluorescence quenching study was conducted. As depicted in Fig. 5A, a fluorescence maxima of PEG-Fmoc alone was recorded at $305 \mathrm{~nm}$ at an excitation wavelength of $270 \mathrm{~nm}$. However, a dramatic fluorescence quenching was observed when PTX was loaded into the micelles, and the extent of decrease in fluorescence intensity was correlated to the amount of PTX loaded in micelles. This fluorescence quenching in the presence of PTX is likely due to the energy transfer caused by the intermolecular $\pi-\pi$ stacking interaction [27] between Fmoc motifs and the aromatic rings of PTX, which may represent the major mechanism of carrier/drug interaction in PEG-Fmoc/PTX (Fig. 1B). As a control, PTX alone did not show any fluorescence at the wavelength range examined.

To further demonstrate that $\pi-\pi$ stacking plays a key role in the Fmoc/PTX interaction, the fluorescence quenching study was similarly conducted with two other hydrophobic molecules, DTX and CHOL (Fig. S4). As depicted in Fig. S5, DTX, an analog of PTX with one fewer aromatic ring, caused slightly less fluorescence quenching, which may be due to a relatively weaker $\pi-\pi$ stacking interaction between DTX and Fmoc. In addition, no significant fluorescence quenching was observed in the hydrophobic interaction between PEG-Fmoc and CHOL, a highly hydrophobic molecule that does not bear aromatic rings. Taking together, these data suggest $\pi-\pi$ stacking as a major mechanism in the PTX/Fmoc interaction. However, hydrophobic interaction and hydrogen bonding may also contribute to the overall interaction between Fmoc and PTX.

Interestingly, PEG-Fmoc-OA exhibited remarkably reduced fluorescence intensity compared to its lipid-free counterpart, PEG-Fmoc, on equal molar basis (Fig. 5B). This severe self-quenching is likely due to the extremely strong carrier-carrier $\pi-\pi$ interaction. Incorporation of lipid chains brought changes to packing parameter and molecular arrangement of PEG-Fmoc-OA assemblies. Unlike PEG-Fmoc that forms spherical micelles, PEG-Fmoc-OA forms filamentous micelles of tubular structures [23]. This morphology alteration may facilitate an overwhelming Fmoc/Fmoc interaction between carriers, leading to an impaired $\pi-\pi$ interaction between Fmoc and PTX. Loading of PTX into PEG-FmocOA micelles may be largely mediated by hydrophobic/hydrophobic interaction. This is in agreement with the finding that loading of PTX into PEG-Fmoc-OA micelles is not associated with dramatic quenching of Fmoc fluorescence. The different modes of drug/ carrier interactions might play a major role in the drastic difference in PTX loading capacity between the two systems (PEG-Fmoc vs PEG-Fmoc-OA).

We further studied the Fmoc/PTX interaction via NMR. Shifts in ${ }^{13} \mathrm{C}-\mathrm{NMR}$ spectra of PTX were recorded in the presence of varying concentrations of PEG-Fmoc. As shown in Fig. 6, PEG-Fmoc caused significant signal shifts in all of the three aromatic rings of PTX, and these signal shifts occurred in an Fmoc concentration-dependent manner. It was also apparent that more dramatic shifts were observed in rings $\mathrm{A}$ and $\mathrm{B}$ than in ring $\mathrm{C}$ as indicated by the average shift index $\triangle$ that represents the average absolute value of the signal shifts of all the six carbon atoms in one aromatic ring. In general, a signal shift of greater than 0.02 in ${ }^{13} \mathrm{C}$-NMR spectrum is believed to be significant and suggestive of intermolecular interaction. These data suggest a strong interaction between Fmoc and the 
aromatic rings of PTX, particularly rings A and B. A stronger interaction between Fmoc and rings A and B of PTX may be attributed to a higher flexibility of the two rings and a less steric hindrance imposed by the bulky core of PTX. The interaction of Fmoc with PTX was similarly demonstrated by NMR with a more simplified system, Fmoc-Lys (Boc)-OH as shown in Fig. S6. The above data shed some insight into the molecular interaction of PEGFmoc with PTX. However, more studies are needed to better understand the mechanism involved in the drastic difference between PEG-Fmoc-OA and PEG-Fmoc with respect to PTX loading capacity.

\subsection{Effect of lyophilization/reconstitution on PEG-Fmoc/PTX mixed micelles}

The preliminary data suggest that PEG-Fmoc may represent as a simple and effective micellar system for delivery of PTX. Therefore, we went on to further characterize PEGFmoc/PTX mixed micelles with respect to their biophysical and biological properties. We first examined the effect of freezing and lyophilization on PEG-Fmoc/PTX mixed micelles since lyophilization is an important procedure for long-term storage in clinical practice. Our results showed that PEG-Fmoc/PTX mixed micelles could be lyophilized and then readily reconstituted in water without any addition of cryo-protectants. In addition, there were no major changes in size distribution following lyophilization and reconstitution (Fig. 7). This property may help to facilitate the clinical investigation of this simple delivery system.

\subsection{Release kinetics of PTX from PEG-Fmoc/PTX mixed micelles}

As an important indicator of formulation stability, release kinetics of PTX from PEGFmoc/PTX was evaluated via a dialysis method, and Taxol, a clinically used PTX formulation $[28,29]$ was employed as a comparison. As depicted in Fig. 8, PEG-Fmoc/PTX mixed micelles exhibited a sustained release profile in PBS, pH 7.4 at $37^{\circ} \mathrm{C}$. After first 24 h, only $19.3 \%$ of entrapped PTX was released from PEG-Fmoc/PTX mixed micelles. Even after $72 \mathrm{~h}$, only $23.5 \%$ of PTX was released from PEG-Fmoc/PTX mixed micelles. This compared favorably to Taxol formulation in which $40.4 \%$ of PTX was released during the $1^{\text {st }} 24 \mathrm{~h}$. The slow kinetics of PTX release is likely due to a strong carrier/drug interaction, which may help to facilitate the PTX delivery through minimizing its premature release from PEG-Fmoc/PTX mixed micelles before they reach the tumor site.

\subsection{In vitro cytotoxicity of PEG-Fmoc/PTX}

In vitro cytotoxicity of PEG-Fmoc/PTX was evaluated with several tumor cell lines including mouse metastatic breast cancer cell line, 4T1.2, and two human prostate cancer cell lines, PC-3 and DU145. As shown in Fig. 9, PEG-Fmoc/PTX mixed micelles exhibited more potent cytotoxicity than Taxol in all the 3 cancer cell lines tested. The carrier alone did not show any significant toxicity to cells under the concentrations tested. This increased cytotoxicity may be attributed to a facilitated entry of PTX into tumor cells. It has been reported that nano-scale delivery systems enter cells through endocytosis [30-32]. This could help to bypass the efflux mediated by drug transporters such as P-glycoprotein, resulting in increased intracellular drug accumulation and improved cytotoxicity. 


\subsection{Biodistribution of PEG-Fmoc/PTX micelles through NIR fluorescent optical imaging}

To investigate the in vivo fate of PEG-Fmoc/PTX, NIR fluorescence imaging was utilized to track the biodistribution of PTX in tumor-bearing mice after i.v. injection of PEG-Fmoc/ PTX. Cy5.5, a near infrared fluorescent probe, was conjugated to PTX and SCID mice bearing human prostate cancer PC-3 xenografts were used. As shown in Fig. 10, Cy5.5-PTX was largely found at tumor sites $24 \mathrm{~h}$ after i.v. administration of PEG-Fmoc/Cy5.5-PTX and substantial amounts of signal remained at tumor sites even $96 \mathrm{~h}$ later. Intravenous administration of free Cy5.5-PTX led to accumulation of the signals largely at the site of kidneys (data not shown). This is largely due to the increased hydrophilicity of PTX following conjugation with Cy5.5, which led to its clearance from the kidneys. After completion of the whole body imaging study at $96 \mathrm{~h}$ post-injection, major organs and tumors were excised, and ex vivo imaging was performed. Strong NIR fluorescence signals of Cy5.5-PTX were found in tumor tissues. Moderate levels of signals were also found in lung and liver, which was due to the nonspecific uptake of nanoparticles by these organs, albeit at relatively low levels [33]. We also noticed significant levels of NIR signals in kidneys, which might be due to the clearance of the Cy5.5-PTX that became dissociated from PEGFmoc/Cy5.5-PTX mixed micelles. Very low NIR levels were found in heart and muscle. It should be noted that coupling of Cy5.5 to PTX led to significant changes in the overall size and other biophysical properties of PTX as reflected by both reduced loading capacity and slightly increased sizes for PEG-Fmoc/Cy5.5-PTX mixed micelles (data not shown). Thus, the targeting efficiency of PEG-Fmoc/PTX might be underestimated with the Cy5.5-PTXbased NIR imaging. The PK/PD study is currently ongoing to directly examine the distribution of PTX in tumors and other major organs/tissues. As a control, Cy5.5-PTX formulated in Cremophor EL yielded poor imaging of the tumor-bearing mice (Fig. S7).

\subsection{Maximum tolerated dose (MTD) study}

The maximum tolerated dose (MTD) of PEG-Fmoc/PTX was examined in tumor-free mice and compared to Taxol formulation. Five different doses of PEG-Fmoc/PTX and three doses of Taxol were tested in BALB/c mice through i.v. injection, followed by monitoring of body weight and other signs of toxicity of these animals. As shown in Table 2, Taxol could be tolerated at a maximum PTX dosage of $20 \mathrm{mg} / \mathrm{kg}$. At this dose, no animal death occurred although several abnormal signs such as convulsion and retarded motion were noticed in most of the treated mice immediately after injection. Compared with Taxol, PEG-Fmoc/PTX exhibited a significantly improved safety profile. Even at a dosage as high as $120 \mathrm{mg}$ PTX/kg (6-fold higher than the MTD of Taxol), neither animal death nor significant weight loss was observed over the entire experiment period. The high MTD of PEG-Fmoc/PTX compares favorably to most of the reported PTX micellar formulations, and is consistent with its excellent formulation stability, slow release profile, and less tendency to accumulate in major organs as illustrated above, which shall provide a much broader therapeutic dosage window for enhanced therapeutic efficacy.

\subsection{In vivo therapeutic efficacy of PEG-Fmoc/PTX}

The in vivo therapeutic efficacy of PEG-Fmoc/PTX was examined in a syngeneic mouse breast cancer model (4T1.2). 4T1.2 is known as a highly metastatic cancer cell line, and as 
demonstrated in Fig. 11A, rapid tumor growth was observed in saline-treated group. Taxol showed a modest effect in inhibiting the tumor growth at a PTX dose of $10 \mathrm{mg} / \mathrm{kg}$. At the same dosage, PEG-Fmoc/PTX was significantly more effective than Taxol in inhibiting the tumor growth $(p<0.05)$. Increasing the PTX dosage was associated with a further enhancement in antitumor activity. At the PTX dosage of 20 and $40 \mathrm{mg} / \mathrm{kg}$, the tumor growth inhibition rate (IR) was 60.6 and 69.7\%, respectively (Fig. 11B). All of the treatments were well tolerated except for a slight decrease of body weight (7-8\%) that occurred following consecutive injections of PEG-Fmoc/PTX at the PTX dose of $40 \mathrm{mg} / \mathrm{kg}$ (Fig. S8). The significantly enhanced antitumor activity and the excellent safety of PEGFmoc/PTX are consistent with its excellent biophysical properties and tumor-selective delivery.

\section{Conclusions}

In summary, a study of Fmoc/PTX molecular interaction has led to the discovery of PEGFmoc conjugate as an effective nanocarrier for PTX. This conjugate can be easily synthesized through a simple three-step route, and it readily forms small-sized $(25-30 \mathrm{~nm})$ micelles with PTX. A strong Fmoc/PTX $\pi-\pi$ stacking interaction provides an excellent drug/ carrier compatibility with a loading capacity $(\sim 36 \%)$ that stands well among the reported micellar formulations. PEG-Fmoc/PTX nanomicelles effectively and selectively accumulated at solid tumors and demonstrated an excellent safety profile with a MTD greater than $120 \mathrm{mg}$ PTX/kg. Finally, PEG-Fmoc/PTX effectively inhibited the tumor growth in a murine breast cancer model, much more effectively than Taxol. Our PEG-Fmoc may hold promise as a simple, safe, and effective delivery system for PTX with a potential for rapid translation into clinical study.

\section{Supplementary Material}

Refer to Web version on PubMed Central for supplementary material.

\section{Acknowledgments}

This work was supported in part by NIH grants R21CA173887, RO1CA174305, and R01GM102989. We would like to thank Dr. Lisa Rohan for her help in DSC study.

\section{References}

1. Ferrari M. Cancer nanotechnology: opportunities and challenges. Nat Rev Cancer. 2005; 5:161-71. [PubMed: 15738981]

2. Farokhzad OC, Langer R. Impact of nanotechnology on drug delivery. ACS nano. 2009; 3:16-20. [PubMed: 19206243]

3. Moghimi SM, Hunter AC, Murray JC. Long-circulating and target-specific nanoparticles: theory to practice. Pharmacol Rev. 2001; 53:283-318. [PubMed: 11356986]

4. Bertrand N, Wu J, Xu X, Kamaly N, Farokhzad OC. Cancer nanotechnology: the impact of passive and active targeting in the era of modern cancer biology. Adv Drug Deliv Rev. 2014; 66:2-25. [PubMed: 24270007]

5. Jain RK, Stylianopoulos T. Delivering nanomedicine to solid tumors. Nat Rev Clin Oncol. 2010; 7:653-64. [PubMed: 20838415] 
6. Riehemann K, Schneider SW, Luger TA, Godin B, Ferrari M, Fuchs H. Nanomedicine--challenge and perspectives. Angew Chem Int Ed. 2009; 48:872-97.

7. Duncan R. Polymer conjugates as anticancer nanomedicines. Nat Rev Cancer. 2006; 6:688-701. [PubMed: 16900224]

8. Torchilin VP. Recent advances with liposomes as pharmaceutical carriers. Nat Rev Drug Discov. 2005; 4:145-60. [PubMed: 15688077]

9. Kedar U, Phutane P, Shidhaye S, Kadam V. Advances in polymeric micelles for drug delivery and tumor targeting. Nanomedicine. 2010; 6:714-29. [PubMed: 20542144]

10. Wagner V, Dullaart A, Bock AK, Zweck A. The emerging nanomedicine landscape. Nat Biotechnol. 2006; 24:1211-7. [PubMed: 17033654]

11. Zhang L, Gu FX, Chan JM, Wang AZ, Langer RS, Farokhzad OC. Nanoparticles in medicine: therapeutic applications and developments. Clin Pharmacol Ther. 2008; 83:761-9. [PubMed: 17957183]

12. Maeda H, Wu J, Sawa T, Matsumura Y, Hori K. Tumor vascular permeability and the EPR effect in macromolecular therapeutics: a review. J Control Release. 2000; 65:271-84. [PubMed: 10699287]

13. Maeda $H$. The enhanced permeability and retention (EPR) effect in tumor vasculature: the key role of tumor-selective macromolecular drug targeting. Adv Enzyme Regul. 2001; 41:189-207. [PubMed: 11384745]

14. Torchilin VP. Structure and design of polymeric surfactant-based drug delivery systems. J Control Release. 2001; 73:137-72. [PubMed: 11516494]

15. Lukyanov AN, Torchilin VP. Micelles from lipid derivatives of water-soluble polymers as delivery systems for poorly soluble drugs. Adv Drug Deliv Rev. 2004; 56:1273-89. [PubMed: 15109769]

16. Gaucher G, Dufresne MH, Sant VP, Kang N, Maysinger D, Leroux JC. Block copolymer micelles: preparation, characterization and application in drug delivery. J Control Release. 2005; 109:16988. [PubMed: 16289422]

17. Huh KM, Lee SC, Cho YW, Lee J, Jeong JH, Park K. Hydrotropic polymer micelle system for delivery of paclitaxel. J Control Release. 2005; 101:59-68. [PubMed: 15588894]

18. Kim JY, Kim S, Pinal R, Park K. Hydrotropic polymer micelles as versatile vehicles for delivery of poorly water-soluble drugs. J Control Release. 2011; 152:13-20. [PubMed: 21352878]

19. Yoon HY, Saravanakumar G, Heo R, Choi SH, Song IC, Han MH, et al. Hydrotropic magnetic micelles for combined magnetic resonance imaging and cancer therapy. J Control Release. 2012; 160:692-8. [PubMed: 22543013]

20. Yoo HS, Park TG. Folate-receptor-targeted delivery of doxorubicin nano-aggregates stabilized by doxorubicin-PEG-folate conjugate. J Control Release. 2004; 100:247-56. [PubMed: 15544872]

21. Yoo HS, Park TG. Folate receptor targeted biodegradable polymeric doxorubicin micelles. J Control Release. 2004; 96:273-83. [PubMed: 15081218]

22. Gao X, Huang Y, Makhov AM, Epperly M, Lu J, Grab S, et al. Nanoassembly of surfactants with interfacial drug-interactive motifs as tailor-designed drug carriers. Mol Pharm. 2013; 10:187-98. [PubMed: 23244299]

23. Zhang P, Lu J, Huang Y, Zhao W, Zhang Y, Zhang X, et al. Design and evaluation of a PEGylated lipopeptide equipped with drug-interactive motifs as an improved drug carrier. AAPS J. 2014; 16:114-24. [PubMed: 24281690]

24. Zhang Y, Gu H, Yang Z, Xu B. Supramolecular hydrogels respond to ligand-receptor interaction. J Am Chem Soc. 2003; 125:13680-1. [PubMed: 14599204]

25. Castelletto V, Moulton CM, Cheng G, Hamley IW, Hicks MR, Rodger A, et al. Self-assembly of Fmoc-tetrapeptides based on the RGDS cell adhesion motif. Soft Matter. 2011; 7:11405-15.

26. Jayawarna V, Ali M, Jowitt TA, Miller AE, Saiani A, Gough JE, et al. Nanostructured hydrogels for three-dimensional cell culture through self-assembly of fluorenylmethoxycarbonyl-dipeptides. Adv Mater. 2006; 18:611-4.

27. Shirai K, Matsuoka M, Fukunishi K. Fluorescence quenching by intermolecular pi-pi interactions of 2,5-bis(N,N-dialkylamino)-3,6-dicyanopyrazines. Dyes Pigments. 1999; 42:95-101. 
28. Rowinsky EK, Donehower RC. Drug-therapy - paclitaxel (taxol). New Engl J Med. 1995; 332:1004-14. [PubMed: 7885406]

29. Singla AK, Garg A, Aggarwal D. Paclitaxel and its formulations. Int J Pharm. 2002; 235:179-92. [PubMed: 11879753]

30. Bertrand N, Leroux JC. The journey of a drug-carrier in the body: an anatomo-physiological perspective. J Control Release. 2012; 161:152-63. [PubMed: 22001607]

31. Vasir JK, Labhasetwar V. Biodegradable nanoparticles for cytosolic delivery of therapeutics. Adv Drug Deliv Rev. 2007; 59:718-28. [PubMed: 17683826]

32. Bareford LM, Swaan PW. Endocytic mechanisms for targeted drug delivery. Adv Drug Deliv Rev. 2007; 59:748-58. [PubMed: 17659804]

33. Sahay G, Alakhova DY, Kabanov AV. Endocytosis of nanomedicines. J Control Release. 2010; 145:182-95. [PubMed: 20226220] 


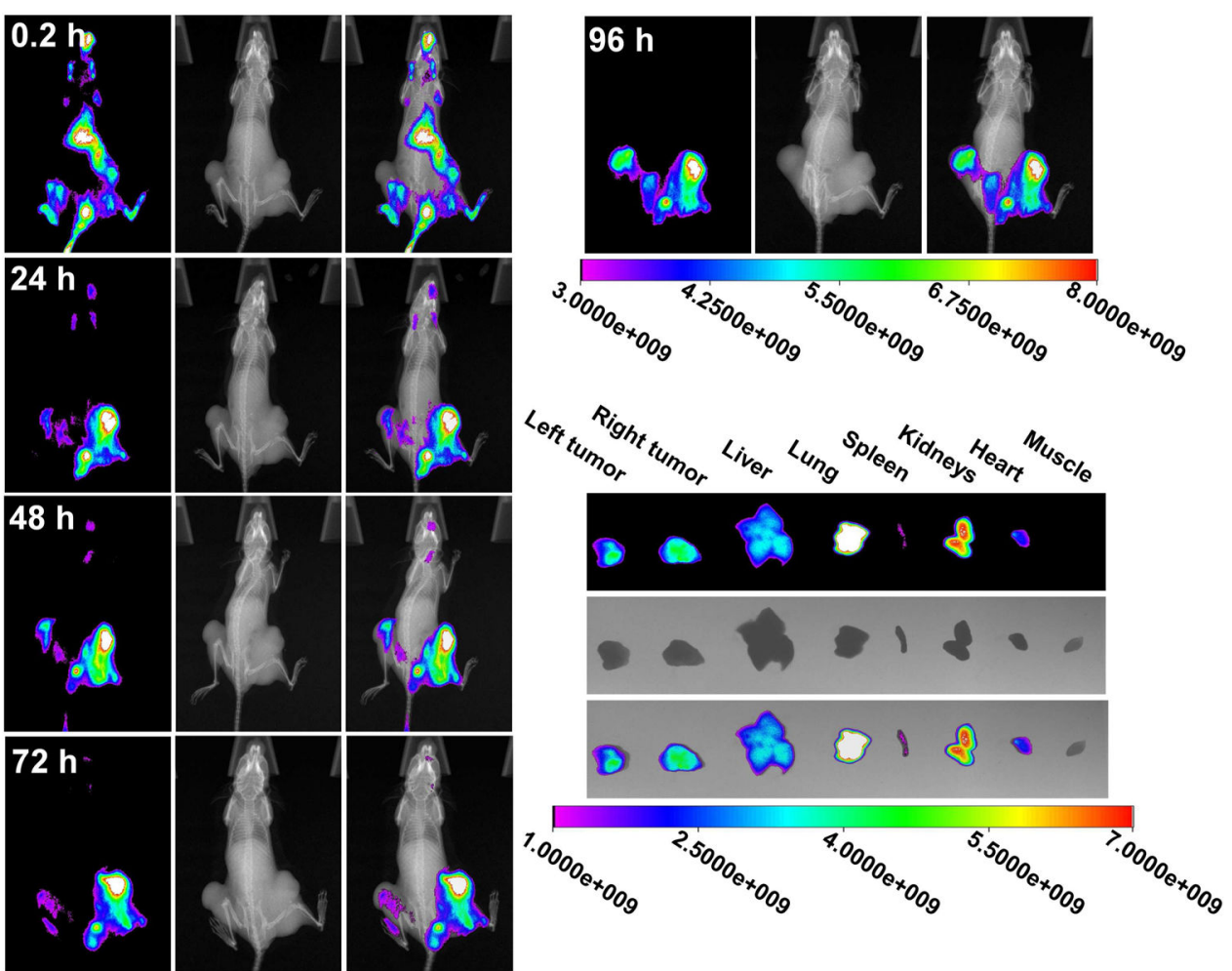

Fig. 1.

Synthetic route of PEG-Fmoc (A), and schematic representation of self-assembled PEGFmoc/PTX mixed micelle based on carrier/drug intermolecular $\pi-\pi$ stacking (B). 


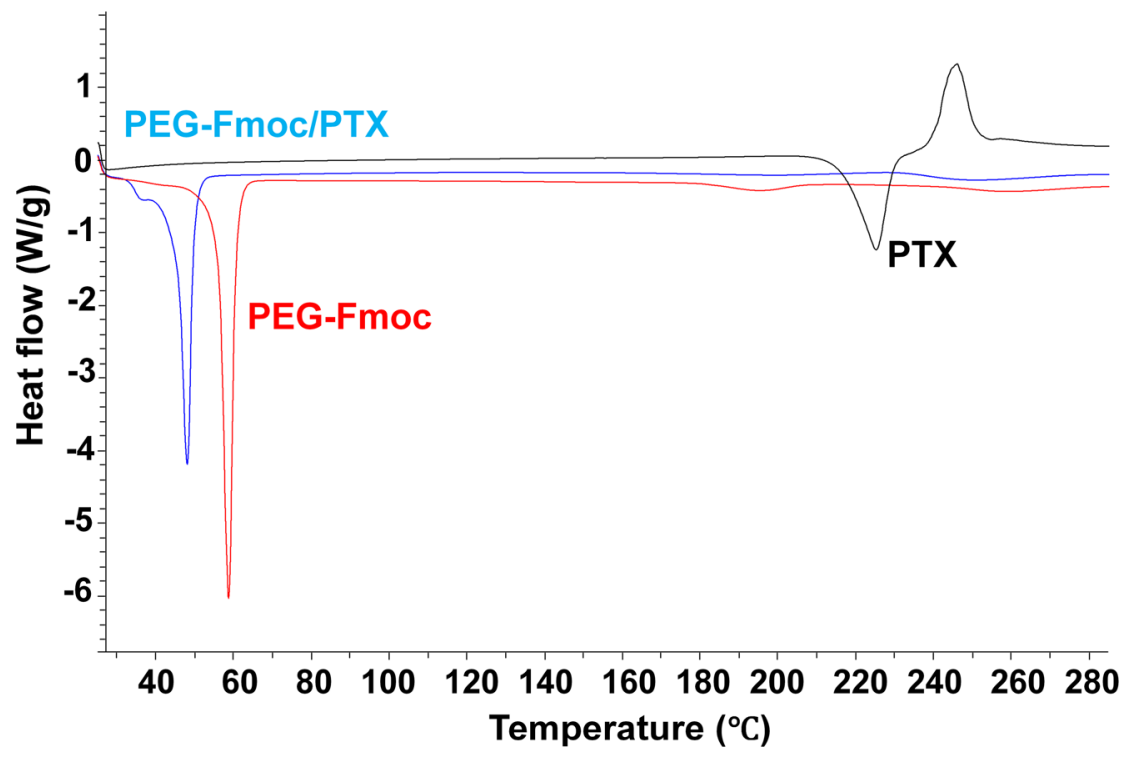

Fig. 2.

Biophysical characterization of PEG-Fmoc nanomicelles. (A) Particle size distribution of PEG-Fmoc/PTX nanomicelles at $1 \mathrm{mg}$ PTX/mL in DPBS with a carrier/drug ratio of 0.75:1 $(\mathrm{m} / \mathrm{m})$ via DLS measurement; (B) TEM image of PEG-Fmoc/PTX nano-micelles with PTX concentration at $1 \mathrm{mg} / \mathrm{mL}$ using negative staining; (C) Critical micelle concentration (CMC) measurement of PEG-Fmoc using pyrene as a fluorescence probe. 

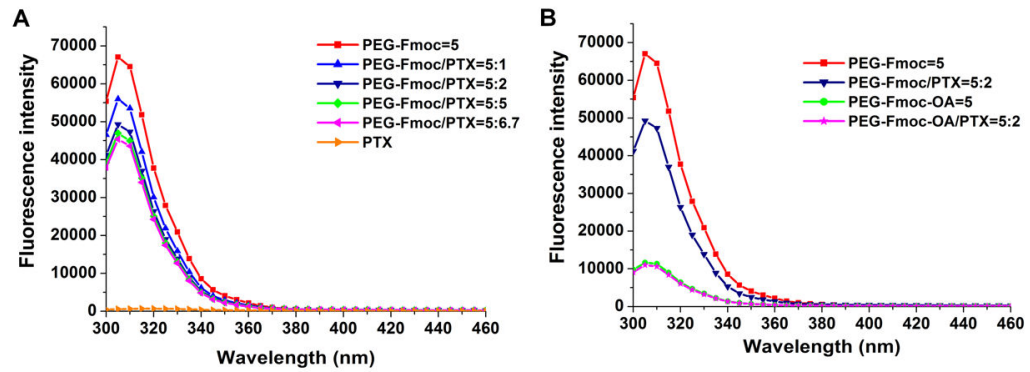

Fig. 3.

${ }^{1} \mathrm{H}-\mathrm{NMR}$ spectra of PEG-Fmoc/PTX in $\mathrm{CDCl}_{3}$ and $\mathrm{D}_{2} \mathrm{O}$. The suppressed proton signals of Fmoc motifs and PTX in $\mathrm{D}_{2} \mathrm{O}$ indicated the self-assembly of PEG-Fmoc micelles in aqueous solution and entrapment of PTX into the interior core of PEG-Fmoc micelles. 


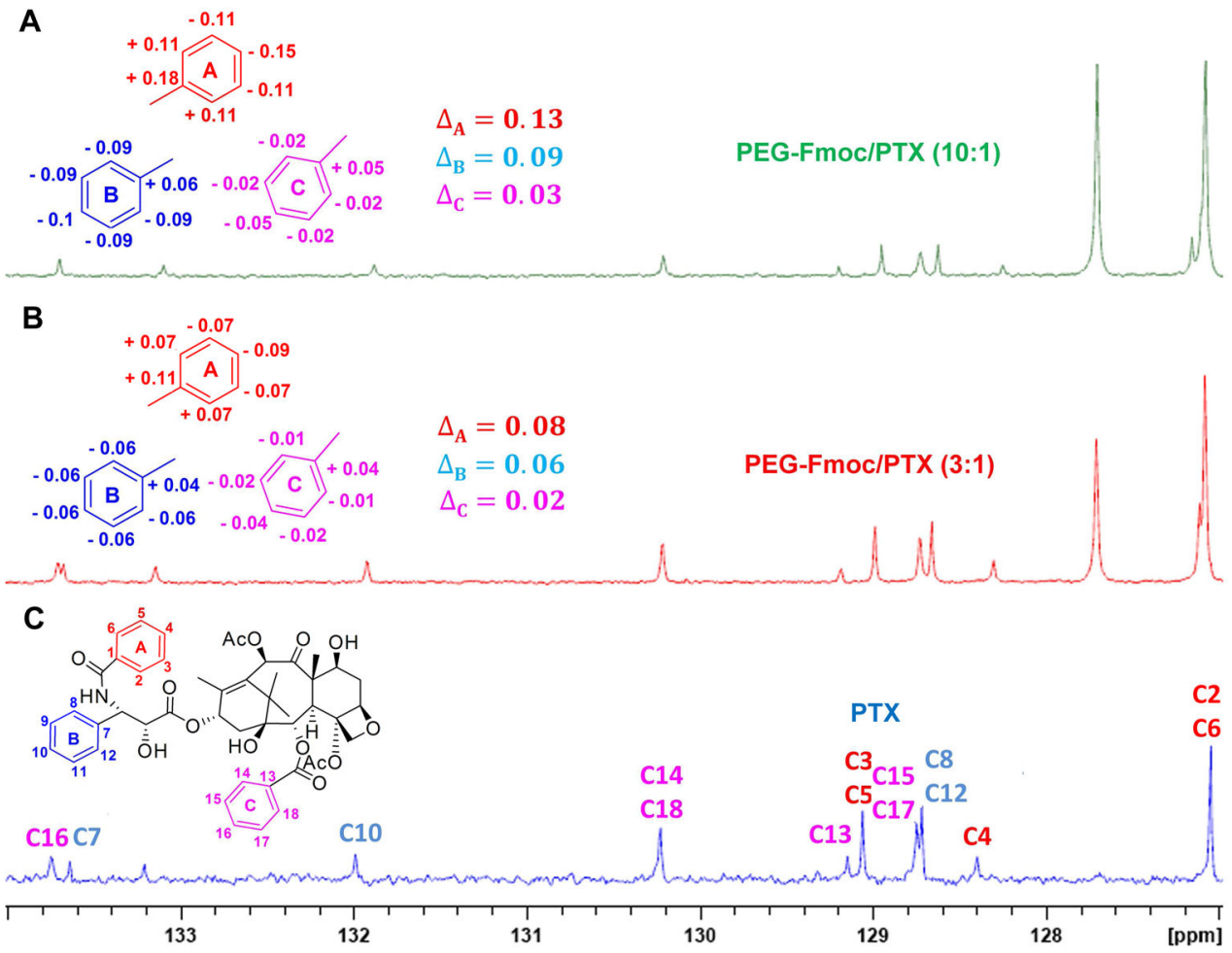

Fig. 4.

DSC thermograms of PTX alone, PEG-Fmoc/PTX mixed micelles, and drug-free PEG-

Fmoc micelles. 


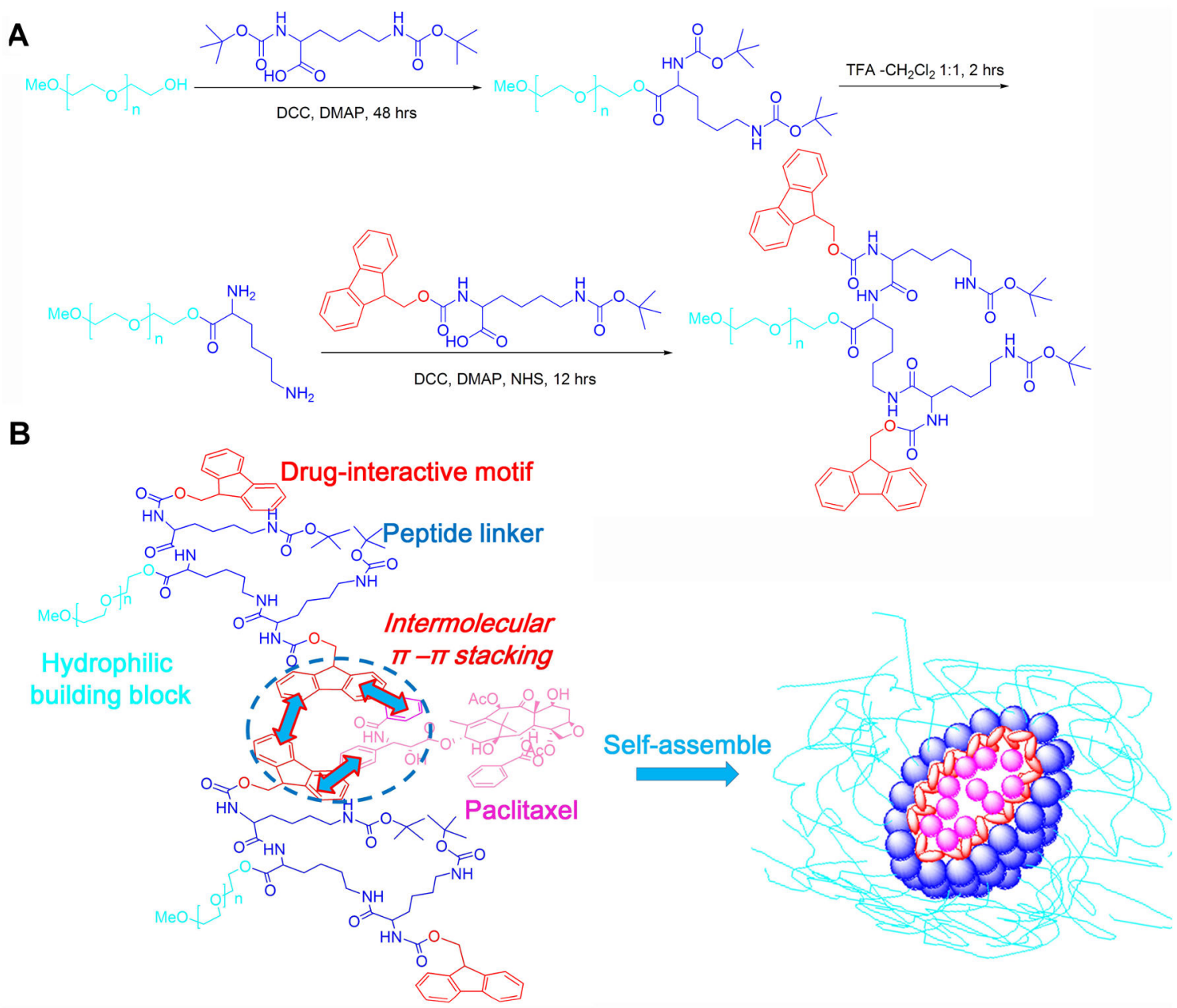

Fig. 5.

Fluorescence quenching study. Fluorescence intensity of PEG-Fmoc (A) at 300- $460 \mathrm{~nm}$ was recorded with an excitation wavelength at $270 \mathrm{~nm}$, and compared with its lipid-bearing counterpart PEG-Fmoc-OA (B). Both PEG-Fmoc and PEG-Fmoc-OA were kept at $0.88 \mathrm{mM}$ for comparison, and all carrier/drug ratios were reported as molar ratios. 
A

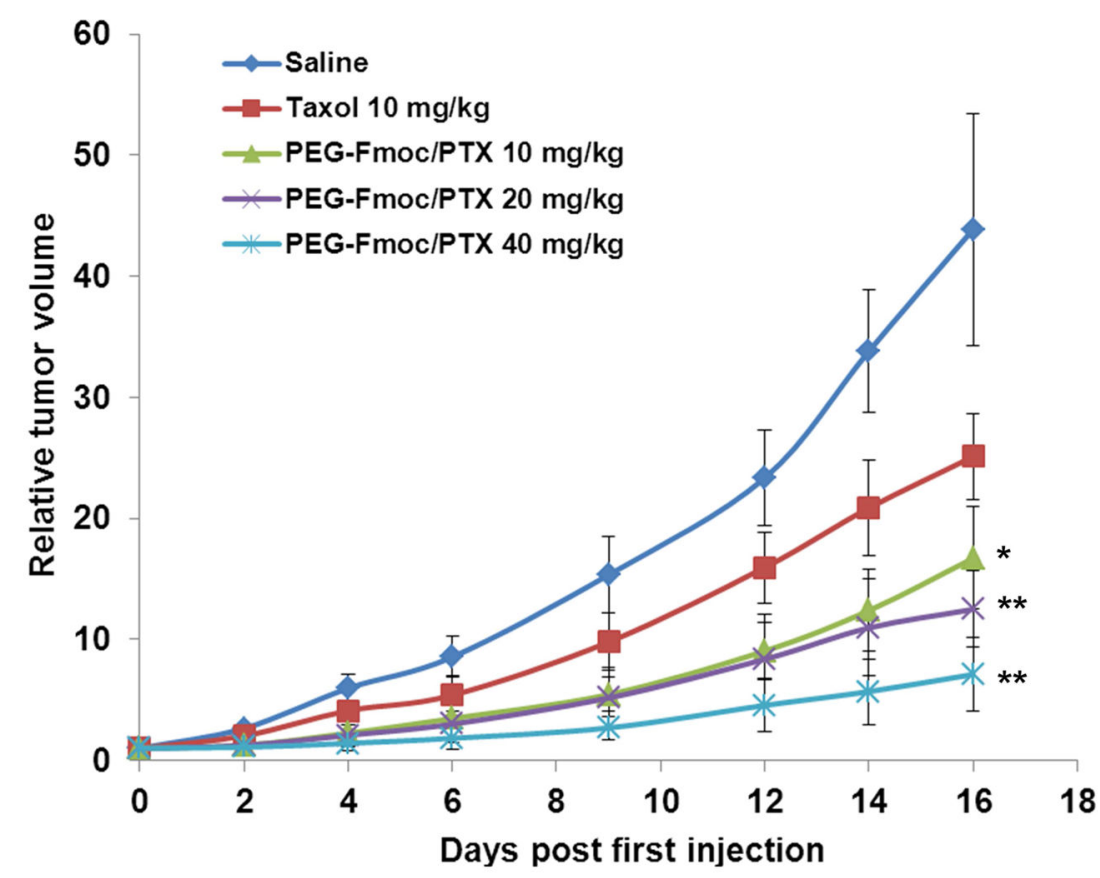

B

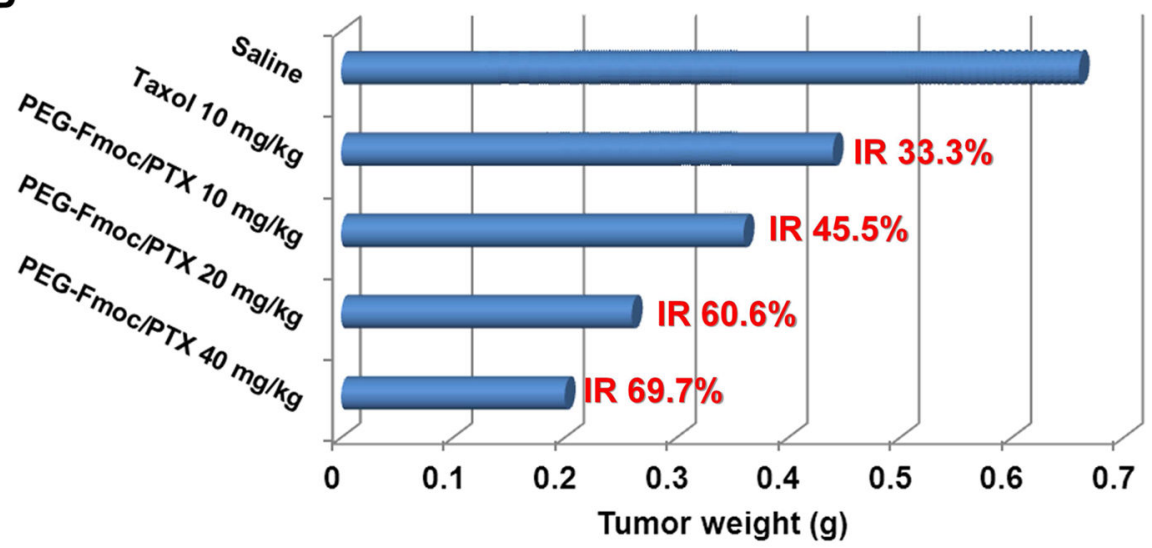

Fig. 6.

${ }^{13}$ C-NMR of carrier-mixed (A \& B) and carrier-free PTX $(\mathbf{C})$ in $\mathrm{CDCl}_{3}$. PTX was mixed with $\mathrm{PEG}_{550}$-a-Fmoc- $\varepsilon$-t-Boc-lysine (see Supplementary data) at a drug/carrier molar ratio of 1:10 (A) and1:3 (B), respectively. Average shift index $(\triangle)$ was calculated as the average absolute value of chemical shift changes of all the six carbon atoms on the same aromatic ring compared with free PTX $(\mathbf{C})$. 

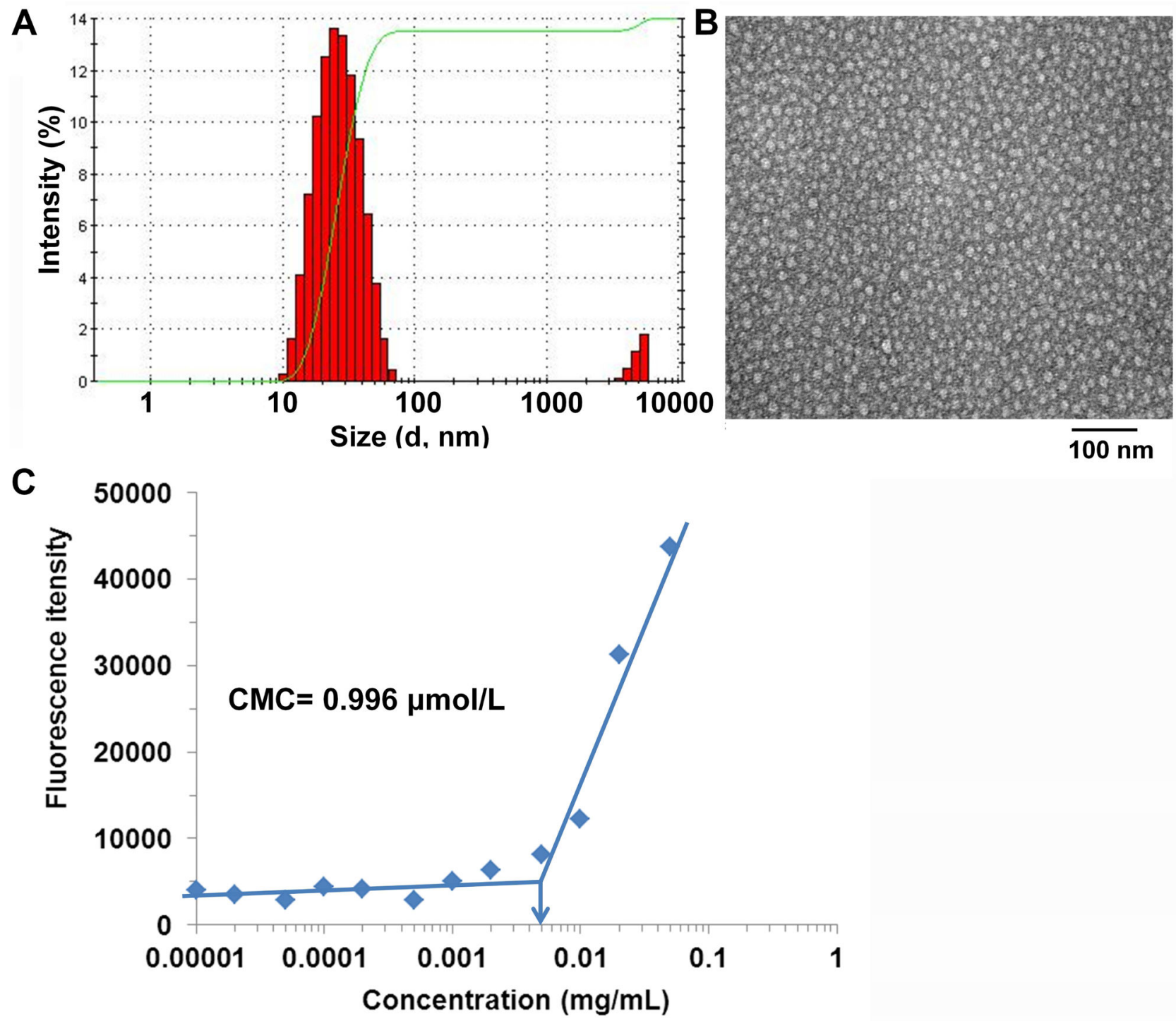

Fig. 7.

Size distribution of PEG-Fmoc/PTX mixed micelles with PTX concentration at $1 \mathrm{mg} / \mathrm{mL}$ before (A) and after (B) lyophilization/reconstitution. 


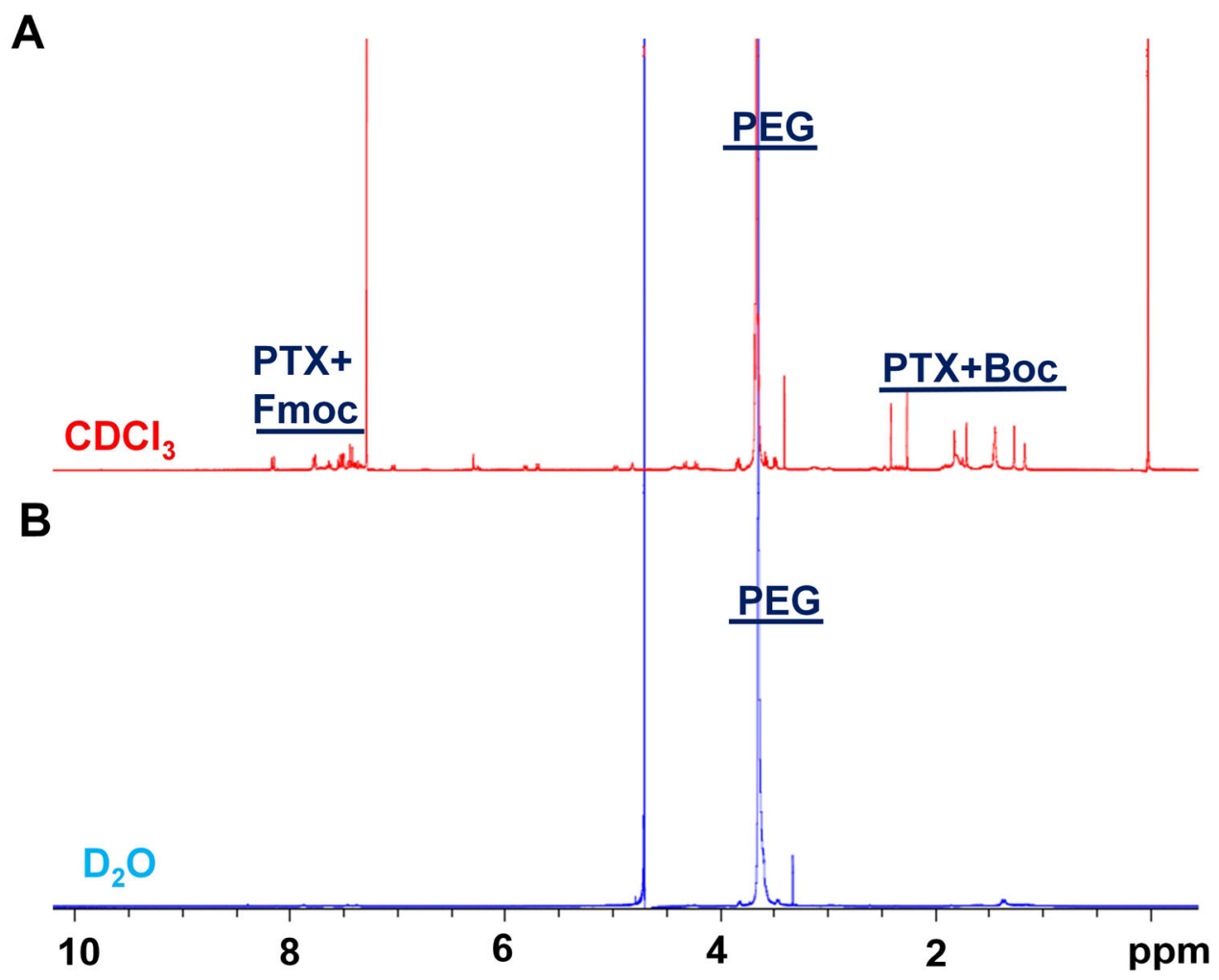

Fig. 8.

PTX release kinetics of PEG-Fmoc/PTX examined via a dialysis method. PTX concentrations in PEG-Fmoc/PTX and Taxol were kept at $1 \mathrm{mg} / \mathrm{mL}$, and DPBS containing $0.5 \%(\mathrm{w} / \mathrm{v})$ Tween 80 was utilized as release medium. PTX concentration was analyzed at 0 , 1, 2, 4, 8, 24, 48 and $72 \mathrm{~h}$ using HPLC at $227 \mathrm{~nm}$. 

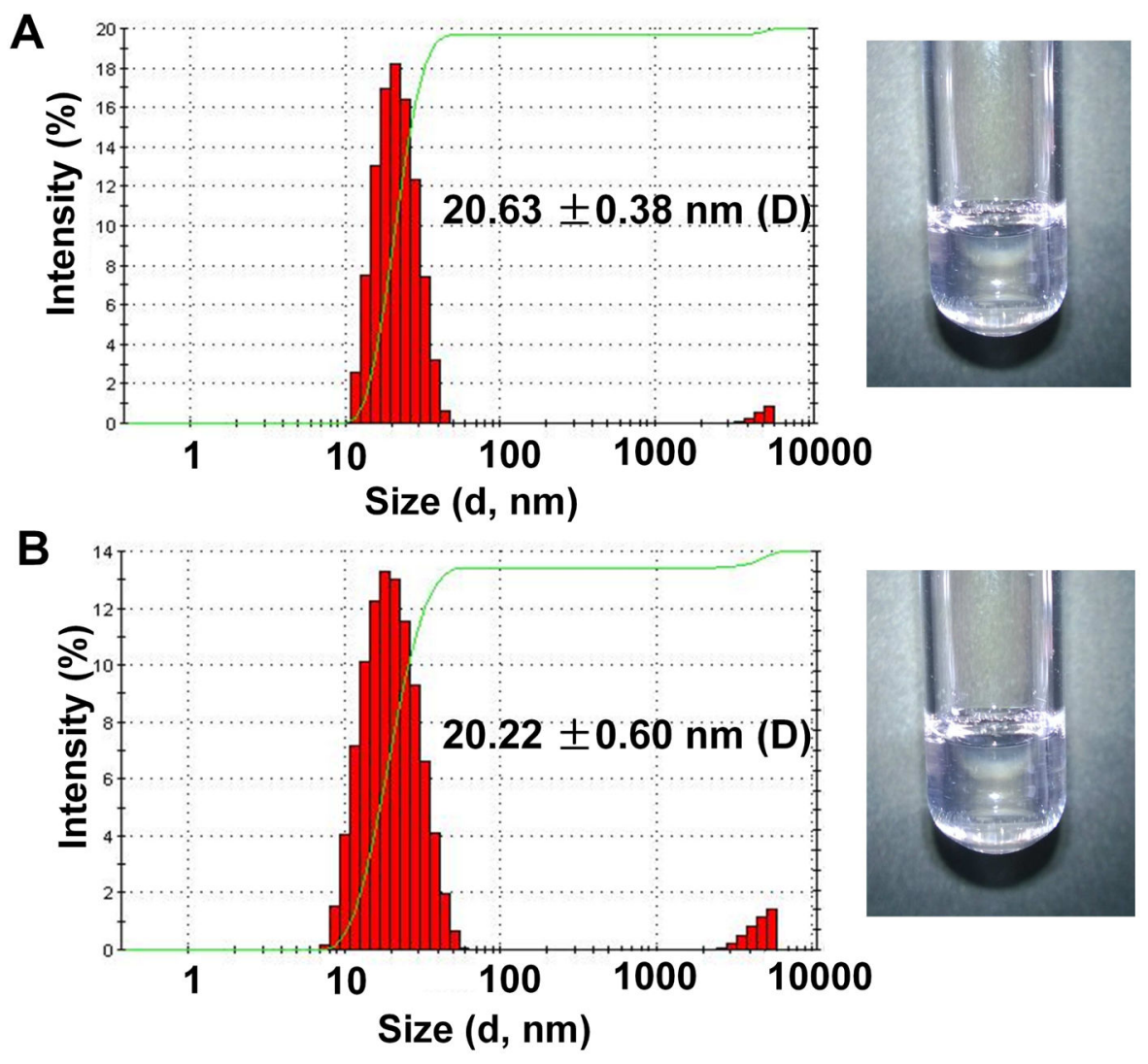

Fig. 9.

In vitro cytotoxicity of PEG-Fmoc/PTX. 4T1.2 mouse breast cancer cell line (A), human prostate cancer cell lines PC-3 (B), and DU145 (C) were treated with PEG-Fmoc/PTX, drug-free PEG-Fmoc and Taxol for $72 \mathrm{~h}$, and tumor cell inhibition was determined by MTT assay. $* P<0.05$ or $* * P<0.01$ (PEG-Fmoc/PTX vs Taxol). 


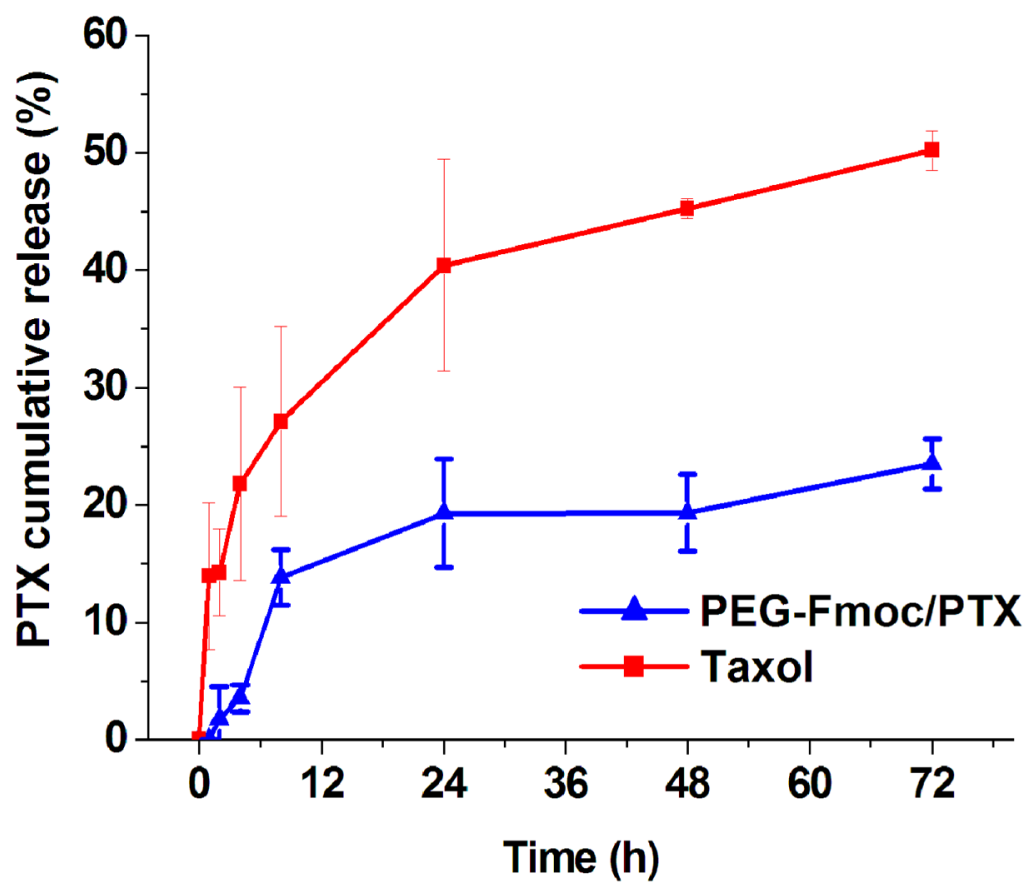

Fig. 10 .

Biodistribution of PEG-Fmoc/Cy5.5-PTX in tumor-bearing mouse as examined by NIR fluorescence imaging. Mouse bearing PC-3 cancer xenograft was scanned at 0.2 h, 24 h, 48 h, 72 h, and $96 \mathrm{~h}$ after i.v. injection of PEG-Fmoc/Cy5.5-PTX; and ex vivo imaging of tumors and major organs was performed $96 \mathrm{~h}$ after injection. 

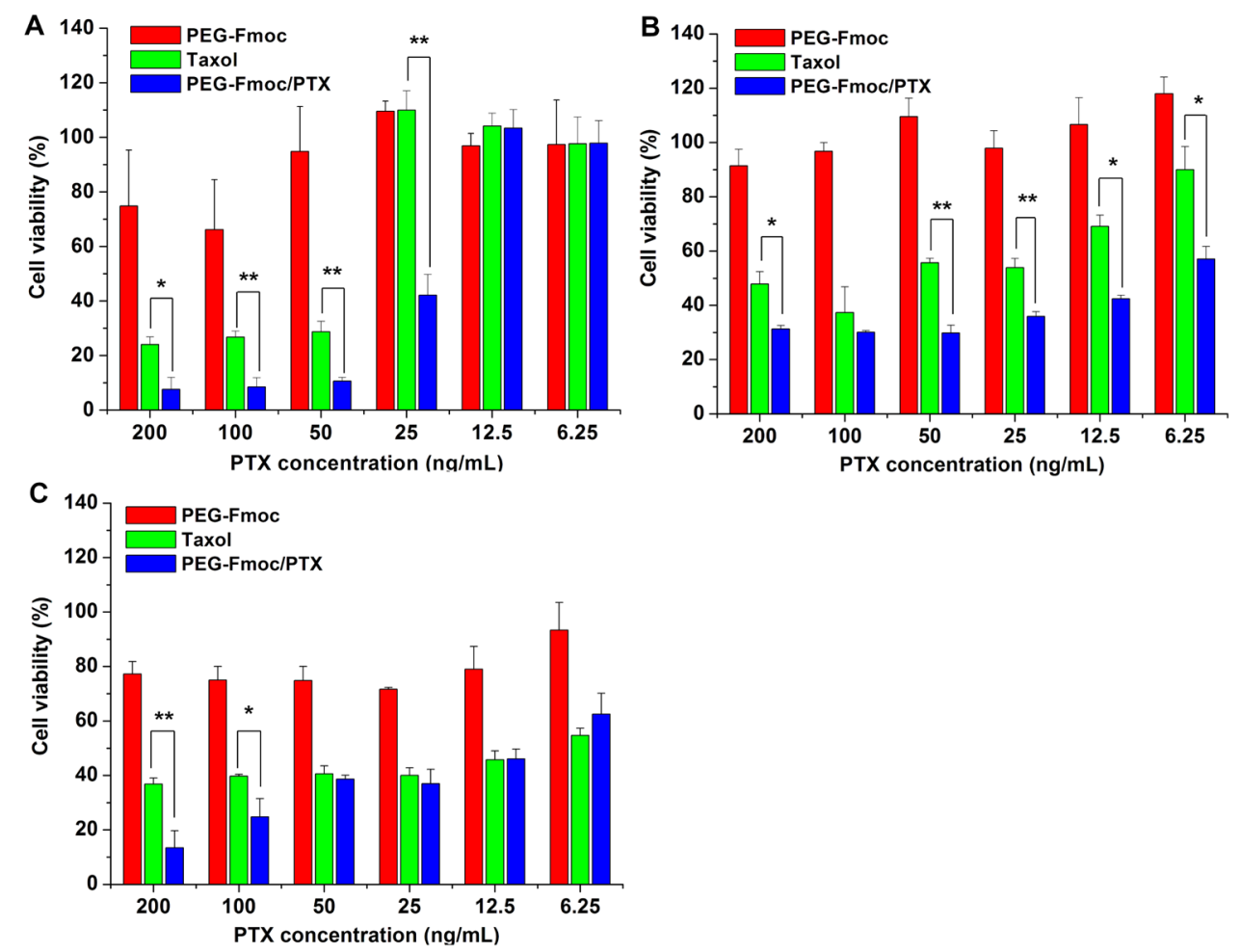

Fig. 11.

In Vivo therapeutic efficacy of PEG-Fmoc/PTX. Groups of 5 BALB/c mice bearing 4T1.2 murine breast cancer grafts were injected with PEG-Fmoc/PTX (10, 20, or $40 \mathrm{mg}$ PTX/kg body weight), Taxol (10 mg PTX/kg body weight) and saline on days $0,2,4,7,10$ and 12, respectively. Tumor growth was monitored and plotted as relative tumor volume (A). ${ }^{*} P<$ $0.05 ; * * P<0.01$ (vs Taxol). Tumor weights were measured after excision and plotted as average tumor weight, and tumor growth inhibition rate (IR) was calculated (B). 


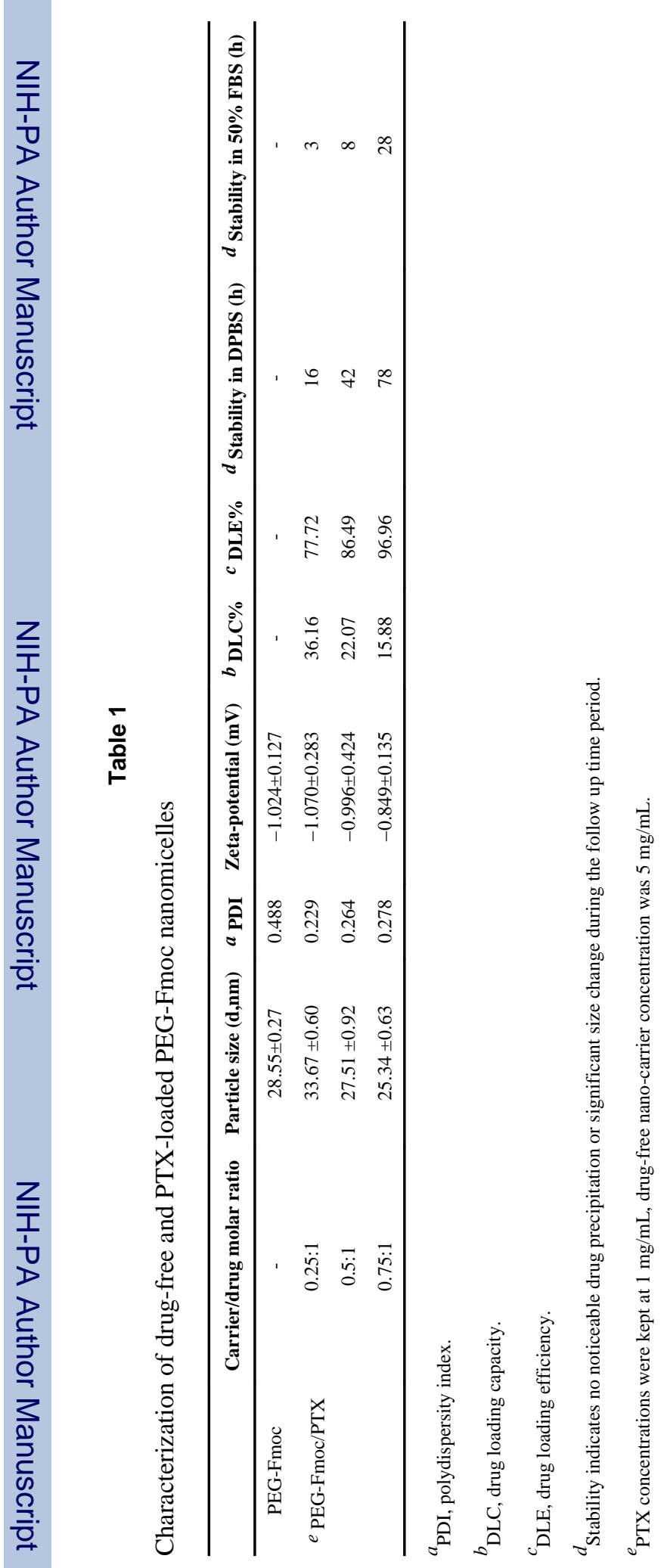

Biomaterials. Author manuscript; available in PMC 2015 August 01. 
Table 2

Maximum tolerated dose of PEG-Fmoc/PTX and Taxol

\begin{tabular}{cccc}
\hline Formulation & Does $(\mathbf{m g}$ PTX/kg) & Mice death & Weight loss $(\%)$ \\
\hline Taxol & 15 & $0 / 4$ & 0.49 \\
& 20 & $0 / 4$ & -4.96 \\
& 25 & $1 / 4$ & -4.35 \\
\hline PEG-Fmoc/PTX & 30 & $0 / 4$ & -2.77 \\
& 50 & $0 / 4$ & -2.82 \\
& 75 & $0 / 4$ & -5.99 \\
& 100 & $0 / 4$ & -5.12 \\
& 120 & $0 / 4$ & -9.49 \\
\hline
\end{tabular}

Biomaterials. Author manuscript; available in PMC 2015 August 01. 